\title{
Relationship of intersession variation in negative pain-related affect and responses to thermally evoked pain
}

\author{
Mark D Bishop, PT, PhD ${ }^{1}$, Jason G Craggs, $\mathrm{PhD}^{2}$, Maggie E Horn, $\mathrm{BS}^{1}$, Steven $\mathrm{Z}$ George, PT, \\ $\mathrm{PhD}^{1}$, and Michael E Robinson, $\mathrm{PhD}^{2}$ \\ ${ }^{1}$ Department of Physical Therapy, University of Florida, Gainesville, Florida, 32610, USA \\ ${ }^{2}$ Department of Clinical and Health Psychology, University of Florida, Gainesville, Florida, 32610, \\ USA
}

\section{Abstract}

The purpose of this study was to determine whether session specific measures of negative pain related affect would account for longitudinal variability in the ratings of the evoked thermal pain. Pain-free subjects rated pain evoked on the posterior leg using thermal stimuli of $45,47,49$ and $51^{\circ} \mathrm{C}$ on three occasions, each separated by two weeks. Session specific negative pain related affect measures were collected at the first session in addition to. Ratings of pain decreased significantly with repeated testing demonstrating a systematic change in rating from the first to second sessions that ranged from a mean of 5.3 at $47^{\circ} \mathrm{C}$ to 9.1 at $49^{\circ} \mathrm{C}$. In addition, large random variation occurred across all sessions resulting in minimal detectable change ranging from 14 to 27 . The least variability occurred when a mean rating of the four temperatures was used. Session specific measures of pain related affect decreased with repeated testing; however the significant between subject variability in both rating of pain and pain-related affect were not related to each other. No associations were identified between psychological measures and variability in rating of evoked pain. Future studies of the variability in ratings should consider other factors such as attentional focus.

Perspective-The individual variability in thermal rating was not explained by individual variation in session specific measures of negative pain-related affect. The results of this study support the use of repeated baseline measures of thermal stimuli when feasible. When this is not possible, the variability in ratings of thermal stimuli over multiple sessions is reduced when the mean of multiple temperatures is used.

\section{Keywords}

fear; threat; challenge; quantitative sensory testing

\begin{abstract}
Quantitative sensory testing (QST) involves the application of standard stimuli to functionally quantify sensory nerve function. Commonly used QST modalities include mechanical, ischemic, chemical and thermal techniques. QST is commonly used to evaluate pain sensitivity. Pain sensitivity is determined when subjects rate their response to the applied stimulus, with elevated pain sensitivity being associated with higher pain responses. Experimental assessment of pain sensitivity has been used to identify factors that influence pain perception in healthy
\end{abstract}

(C) 2009 The American Pain Society. Published by Elsevier Inc. All rights reserved.

Corresponding Author: Mark Bishop, PO Box 100154, Gainesville, Fl 32610-0154, USA, (352) 2736112, (352) 2736109 bish@ ufl.edu. Publisher's Disclaimer: This is a PDF file of an unedited manuscript that has been accepted for publication. As a service to our customers we are providing this early version of the manuscript. The manuscript will undergo copyediting, typesetting, and review of the resulting proof before it is published in its final citable form. Please note that during the production process errors may be discovered which could affect the content, and all legal disclaimers that apply to the journal pertain. 
subjects, such as sex 8,9 and age differences.5, 11 Also, recent cross-sectional studies indicate individuals with chronic pain syndromes demonstrate increased pain sensitivity when compared to healthy subjects. Increased pain sensitivity is consistently reported in patients with fibromyalgia, $27^{-} 29$ pelvic floor pain, ${ }^{12}$ temporomandibular disorders (TMD) ${ }^{24}$ and patients with chronic low back pain.14

Experimental pain responses to QST can be used as predictors of outcome following interventions that may cause pain. For example, pressure pain thresholds taken before limb amputation are inversely correlated with post-amputation pain in the residual limb and phantom pain. ${ }^{18}$ Additionally, pre-operative thermal hyperalgesia was shown to be associated with pain after total knee arthroplasty, and was predictive of postoperative morphine consumption. ${ }^{16}$ QST may also predict favorable response to interventions meant to reduce pain. Schiff et $\mathrm{al}^{25}$ report that specific responses to QST were associated with favorable responses to epidural steroid injections in patients with sciatic pain indicating that QST might act as a tool in the selection of the appropriate treatment for these patients; that is in this case, injection versus surgery.

Responses to QST can also be used as the outcome measure to document intervention-related changes in pain sensitivity. Indeed, Edwards et al ${ }^{6}$ have suggested QST measure may be a sensitive index of treatment outcome given that "treatment-related normalization of pain perception is observed only when clinical pain is reduced." For example, thermal and mechanical hyperalgesia in patients with osteoarthritis is reduced following successful painrelieving surgery 15 and subjects with lateral epicondalgia experience reductions in pressure pain threshold after a manual therapy intervention to the cervical spine. ${ }^{7}$ In our lab we have shown that changes in response to thermal QST occur acutely after manual therapy intervention to the lumbar spine. ${ }^{1,10}$

This use of QST to predict outcome or to follow subjects over time suggests that responses to QST are consistent in both subjects without and with pain. However, some evidence suggests that this may not be this case, at least in subjects without pain. Indicative of this, Yarnitsky et al34 reported large coefficients of reliability across testing sessions in subject-specific responses to thermal stimuli presented in set increments above the thermal pain threshold. However, Rosier et al23 suggested this to be a problematic design as thermal threshold was also shown to be quite variable thereby compounding the variability. In their own study these authors attempted to "distinguish session-to-session variations in pain perceptions from session-to-session variations in measurements of those perceptions." 23 They concluded that variation in the manner in which subjects used the pain rating scale at each session occurred as well as variation in the pain experience for each subject. Recently published work also indicated small but significant decreases over time in ratings of pain associated with thermal stimuli and these authors investigated whether individual attributes such as trait positive or negative affect, anxiety, or sex influenced the variability in ratings. ${ }^{21}$ Their primary finding was that while pain intensity negatively correlated with the variation in rating (the lower a subject rated a stimulus the greater the variability across- and within-testing sessions) individual attributes were not consistently associated with the variation.

This current paper, therefore, had the overall goal of extending previous studies that examined variability in rating of thermal stimuli by collecting session specific negative pain-related affect (for example, anxiety about potential pain resulting from the experimental stimuli). We hypothesized that variability in session specific negative pain-related affect would account for variability in the ratings of the evoked thermal pain. 


\section{METHODS}

\section{Subjects}

Healthy volunteers were recruited from the general student and faculty population at the University. Power calculations from a preliminary study of twelve subjects indicated that a sample of 29 was needed for significant association between affect and rating of $51^{\circ} \mathrm{C}$ at Session 1 , and 32 subjects for change in rating from Session 1 to Session 3 at $51{ }^{\circ} \mathrm{C}$. All the participants reported that they had been free of musculoskeletal pathology for at least six months prior to participation. Participants were counseled not consume caffeine two hours before each testing session and had to be able to attend all sessions. The study design and protocol were approved by the University Institutional Review Board and each subject read and signed an informed consent form. After the consent process, subjects completed a demographic survey.

At each testing session, subjects completed session specific measures of negative pain-related affect and provided pain ratings in response to thermal QST. There were three testing sessions separated by approximately two weeks.

Session specific measures-Measures of negative pain related affect associated with the potential pain of thermal QST were measured using $100 \mathrm{~mm}$ VAS. Specific statements were administered using "none" and "worst imaginable" provided as scale anchors. These statements were:

Describe your current level of fear about any pain you are about to feel.

Describe your current level of anxiety about any pain you are about to feel.

Describe how threatened you are by any pain you are about to feel.

Describe how challenged you are by any pain you are about to feel.

The measures were collected prior to thermal testing at each session. The threat VAS and challenge VAS measures have been previously associated with intensity of pain from thermal stimuli $^{4}$ and a anxiety VAS measure predicted reports of pain in trials studying placebo. ${ }^{31}$

Intensity of thermally evoked pain was also rated using a $100 \mathrm{~mm}$ visual analog scale (VAS) anchored at one end with 'none' or and at the other with 'worst imaginable.' Subjects rated pain by placing a mark along the $100 \mathrm{~mm}$ line. A previous study has indicated that the VAS is a valid ratio measure for pain intensity. ${ }^{19}$

Thermal QST_During each visit, subjects rode a Monarch Bicycle Ergometer (MonarchCrescent AB, Varberg, Sweden) for 5 minutes at $1 \mathrm{kP}$ and 55rpm to standardize the level of activity performed by the subjects immediately prior to each testing session.

All thermal stimuli were delivered to the skin of subjects using a computer-controlled Medoc Neurosensory Analyzer (TSA-2001, Ramat Yishai, Israel). Stimulation sites were varied to prevent carryover effects due to local sensitization. We had both a male and female examiner present during testing to account for sex and/or gender influence on pain reporting. ${ }^{22}$

Before every testing session, subjects underwent a practice session. During this session subjects experienced the temperatures to which they were to be exposed. Subjects practiced using the rating scale to rate the intensity of the first pain experienced in response to each stimulus. In order to standardize the scaling instructions and to clarify the distinction between the sensory intensity and affective dimensions, a standardized instructional set was used for all subjects during every exposure to the thermal stimuli. The scale instructions were repeated for every set of ratings within each session. ${ }^{23}$ 
After the practice session, heat stimuli of 5 seconds duration were applied to the posterior surface of the upper calf below the popliteal fossa, with the subject in sitting. The subjects experienced a sequence of four thermal pulses that included $45,47,49$, or $51^{\circ} \mathrm{C}$ presented randomly. Subjects were cued to provide a rating of any pain experienced immediately after the peak of each thermal pulse. This procedure was performed twice. The interstimulus interval was at least 60 seconds to avoid carryover effects from one stimulus to another, to prevent changes in receptor responses and to prevent tissue changes. Temperature levels were monitored by a contactor-contained thermistor, and returned to a preset baseline of $35^{\circ} \mathrm{C}$ by active cooling at a rate of $10^{\circ} \mathrm{C} / \mathrm{sec} .^{27,33}$

\section{Statistical analysis}

Sex differences in measures taken at baseline were tested using the Mann-Whitney U test.

To examine whether 1) differences in ratings occurred from the first to second exposure of the same temperature within a single session and 2) differences occurred between sessions, we performed separate two-way repeated measures analysis of variance (ANOVA) with factors for session (three levels), and exposure (first and second application of each temperature) for each temperature. Additionally ratings of the thermal stimuli were averaged across stimulus temperatures to yield a mean rating for each subject $(\mathrm{AvP})$ and the analysis was repeated. Sex was added as a between subjects factor for any temperature at which there sex differences in rating noted. Follow-up one-way ANOVA or t-tests were performed as appropriate using Bonferonni corrections to maintain type 1 error at $5 \%$.

Minimal detectable change (MDC) in pain ratings across the sessions was estimated for each temperature and AvP. Random changes in rating were assessed by calculating the standard error of measurement (SEM) using the square root of the mean square error term from a oneway random effects model ANOVA. ${ }^{30}$ MDC was calculated using 1.96*SEM. Additionally any systematic change (bias) for the group was determined by calculating the average change score between pairs of sessions that differed significantly from each other.2

Data from any of the VAS measures of fear, anxiety, threat, and challenge that showed significant change over testing sessions were used to predict first pain ratings to thermal stimuli by applying a multilevel modeling (MLM) approach. MLM, also referred to as hierarchical linear modeling, HLM, ${ }^{3}$ is an extension of the general linear model and does not require observations to be independent. Thus, MLM is a very flexible tool that is well suited for repeated data, given their autoregressive nature and hierarchical structure (i.e. observations nested within each participant). 26,32,35

Fixed (group) and random (individual) effects can be estimated with MLM. The benefit of the random-effects analysis is that it avoids the $d f$ inflation that occurs as a result of repeated sampling per subject. Fixed effects refer to "average effects", or effects that hold for all members of the group. Random effects test whether there are significant individual differences in obtained fixed effects. For example, a significant random variance term would indicate that the magnitude of that within-person relationship may differ substantially across individuals.

All analyses were performed using SPSS 15.0 for Windows (SPSS Inc., Chicago, USA). Type 1 error was maintained at $5 \%$ for each analysis.

\section{Results}

Thirty-five subjects participated in this study (aged 24.3 $\pm 2.9 ; 26$ females, ten males). Females rated $45^{\circ} \mathrm{C}$ higher than males (Table 1) therefore sex was added as a factor to the analyses of $45^{\circ} \mathrm{C}$. 


\section{Within session differences}

No significant interactions were noted at any temperature indicating that no differences occurred between the first and second exposure of each temperature that were specific to a particular testing session. However, there was a significant main effect at $45^{\circ} \mathrm{C}\left(\mathrm{F}_{1,68}=5.74\right.$, $\mathrm{p}=0.022$ ) in which the second presentation of $45^{\circ} \mathrm{C}$ was rated lower than the first. This effect was not noted for any other temperature, nor was it noted for AvP. Sex was not a significant factor as a main effect or in any interactions ( $p>0.05$ for all).

\section{Across session differences}

Significant main effects for session were noted for each temperature and for the AvP. This effect was consistent in that the ratings at Session 2 were lower than Session 1, but Session 2 was not different from Session 3. Figure 1 illustrates the variation in rating both within and across testing sessions.

\section{Minimal detectable change in ratings of pain between sessions}

The MDC across all sessions was calculated for pain ratings $(0-100)$ of individual temperatures ranged from 24 to 30 . When calculations were repeated based only the ratings of session 2 and session 3, the MDC was slightly reduced ranging from 18 to 26. The MDC of the AvP was smaller than that when individual temperature ratings were considered (17 and 14 respectively).

Statistical differences in the ratings of stimuli were identified between Session 1 and Session 2 at all temperatures and AvFP suggesting systematic bias in these ratings. ${ }^{2}$ Examination of the confidence intervals around the point estimates of systematic bias showed that statistically significant bias occurred for ratings at $45^{\circ} \mathrm{C}, 49^{\circ} \mathrm{C}, 51^{\circ} \mathrm{C}$ and $\mathrm{AvP}$. The results are summarized in Table 2.

\section{Predicting variation in rating using session specific measures of pain related affect}

To examine measures of pain related affect as candidates for predicting pain rating variability across time, each pain-related affect variable was examined in a univariate analysis. The amount of perceived challenge of testing decreased over time $\left(\mathrm{F}_{1,34}=12.6, \mathrm{p}=0.001\right)$, while the threat of testing remained unchanged. Both fear and anxiety decreased with repeated exposure to the thermal testing $\left(\mathrm{F}_{1,34}=6.8, \mathrm{p}=0.013\right.$ and $\mathrm{F}_{1,34}=4.3, \mathrm{p}=0.047$ respectively). Thus session specific challenge, fear, and anxiety were used during MLM.

For the MLM, ratings in response to thermal stimuli were averaged across stimulus temperatures to yield a mean rating (AvP) for each participant at each time point. These values were used as the dependent variable. The only findings of the MLM modeling were that the random effect for fear $(\mathrm{p}<0.001)$ and individual variation in $\mathrm{AvP}(\mathrm{p}<0.022)$ were significant. That is, both individual variation in fear from session to session and individual rating of thermal pain each time point was different among individuals. However, affective variability and AvP variability were not significantly related to each other.

\section{Discussion}

These findings indicate that ratings of thermal stimuli are expected to decrease over time for healthy subjects. The decrease varies from individual to individual and is not related to session specific measures of pain-related affect. This potentially provides an important caveat to use of thermal QST as an outcome measure. The first step toward determining whether an intervention has affected thermal pain sensitivity is to determine whether any change in rating from session to session exceeds the boundaries of the normal variation in the measure. 
MDC in the ratings of thermal stimuli ranged from 13.7 to 29.2. These values were dependent on the temperature of the stimulus and whether Session 1 was included in the calculations. Use of values from Session 1 was also problematic due to the presence of the systemic change or bias found from Session 1 to Session 2. For example, a subject who rated $49^{\circ} \mathrm{C}$ as $50 / 100$ at Session 1 might be expected to rate the same stimulus between 21/100 and 79/100 at a second session. Thus an intervention proposed to cause hypoalgesia would potentially need to cause a reduction in rating greater than $50 \%$ of the one taken at Session 1 .

The MDC interval narrows somewhat using the calculations based on Sessions 2 and 3 as the SEM is smaller and no systematic bias was noted. In this case, if the subject rated $49^{\circ} \mathrm{C}$ as 50 on the pain scale, a change beyond random variation at Session 3 would need to be outside of the interval bound by 32/100 and 68/100. The smallest MDC was created using the mean of the ratings at the four different temperatures. A subject with AvFP of 50 at Session 2 is likely have an AvP between 36/100 and 64/100 at the subsequent testing session.

While these results are specific to this sample and subject to the inherent limitations of sample size, the large MDC values are consistent with the wide confidence intervals at individual reported by Yarnitsky et al. ${ }^{34}$ Our data suggest that using the mean rating of the four temperatures might provide more reliable ratings of thermal stimuli than ratings of the individual temperatures. Additionally the finding of systematic change from Session 1 to Session 2, in all measures, suggests a learning effect. Given that our practice session occurred on the same day as the first testing session, these data indicate that a separate testing session for the practice and familiarization session is indicated prior to beginning baseline data collection.

Changes in how a subject might rate thermally-evoked pain from session to session can have multiple causes. For example, psychological variables contribute to the individual experience of pain. ${ }^{20}$ Quinton and Greenspan ${ }^{21}$ tested association between the coefficient of variation in rating and state anxiety and negative and positive affect and reported no consistent associations. We chose to build on this previous work and test whether session specific measures taken at each testing session might influence the rating of thermal pain within that session. We chose measures that had previously been associated with thermal pain. ${ }^{4}$ While fear, anxiety and challenge did vary across testing sessions, variation in these measures did not explain variation in the ratings of thermal pain. Instead, pain-related affect varied in both in direction and magnitude across individuals from session to session. This was also the case for ratings of the thermal stimuli but the variation was not consistent with pain related affect. For example, of the subjects tested, four increased their rating of the thermally evoked pain from session to session while five others increased at session 2 and then returned to the baseline at session 3 .

Other causes of changes in the experience of thermally-evoked pain include physical and physiological variables. We attempted to control physical variables as much as possible by testing the same laboratory room and on the same computer controlled device and the stimulus sites were consistent from session to session. All subjects were advised to avoid caffeine or any pain medications prior to testing. All subjects also performed a standardized bout of activity prior to testing in attempt to control for prior activity levels.

Consequently, the fluctuations in rating are likely related to other aspects of the daily experiences of an individual that were not accounted for with our methodology. We could not account for the experiences of each subject in their personal life, for example, or their level of arousal on that particular day. Over the repeated sessions, the interpersonal relationship between subject and testers may have changed and subjects may have become more relaxed about the experience of the experiment which may not have been directly reflected in their responses about session specific pain-related affect. Also, although we used standard 
instructions about using the pain scale at each testing session, we did not standardize instructions regarding attention to the stimuli. Subjects may have varied in the amount of attention to the experience of the stimuli from session to session. Literature indicates that increased attention is associated with increased reports of pain ${ }^{13,17}$ and decreasing this attention may assist in decreasing pain suggesting that manipulating attention in experimental induced pain will likewise modify the pain reporting of the subjects. ${ }^{17}$ Further investigation is required to determine if specific instructions regarding attentional focus will minimize the variability of ratings of thermally-evoked pain when measured longitudinally or whether other stress factors can be controlled or measured.

We did not account for hormonal variation or take specific physiological measurements prior to testing (heart rate and blood pressure, for example). Consequently we are unable to comment on the potential for these physiological variables to predict variation in reporting of thermal pain. Nor did we collect measures of sympathetic arousal which may have varied among subjects with similar levels of fear of pain.

Additionally, our data do not provide an estimate of when or if the ratings of thermal pain stabilize. In fact, this individual variability may make it difficult to develop a definition for when stability occurs in this measure. Consistent with previous literature, a practice session that is separate from the first data collection is warranted; while this will not eliminate individual variation, it may help reduce it. These measures may not be appropriate for repeated testing of a single group over time. Instead, experimental designs that include a control group seem more appropriate so that the variability in responses is accounted for equally in experimental conditions. Our data also suggest that the average of ratings at multiple temperatures might be a more stable and reliable measurement in subjects not experiencing pain. However, reliance on such an average value may reduce information about the individual variation at each stimulus intensity.

\section{Acknowledgments}

This project was supported by funding from the National Institute of Arthritis and Musculoskeletal and Skin Diseases and the National Center for Complementary and Alternative Medicine.

\section{References}

1. Bialosky JE, Bishop MD, Robinson ME, Barabas JA, George SZ. The influence of expectation on spinal manipulation induced hypoalgesia: an experimental study in normal subjects. BMC Musculoskelet Disord 2008;9:19. [PubMed: 18267029]

2. Bland JM, Altman DG. Measuring agreement in method comparison studies. Stat Methods Med Res 1999;8(2):135-160. [PubMed: 10501650]

3. Bryk, AS.; Raudenbush, SW. Hierarchical linear models for social and behavioral research: Applications and data analysis methods. Newbury Park, CA: Sage; 1992.

4. Dannecker EA, Price DD, O'Connor PD, Robinson ME. Appraisals of pain from controlled stimuli: relevance to quantitative sensory testing. Br J Health Psychol 2008;13(Pt 3):537-550. [PubMed: 17681110]

5. Edwards RR, Fillingim RB. Effects of age on temporal summation and habituation of thermal pain: clinical relevance in healthy older and younger adults. J Pain 2001;2(6):307-317. [PubMed: 14622810]

6. Edwards RR, Sarlani E, Wesselmann U, Fillingim RB. Quantitative assessment of experimental pain perception: multiple domains of clinical relevance. Pain 2005;114(3):315-319. [PubMed: 15777856]

7. Fernandez-de-las-Penas C, Perez-de-Heredia M, Brea-Rivero M, Miangolarra-Page JC. Immediate effects on pressure pain threshold following a single cervical spine manipulation in healthy subjects. J Orthop Sports Phys Ther 2007;37(6):325-329. [PubMed: 17612359]

8. Fillingim R. Sex, gender and pain: women and men really are different. Curr Rev Pain 2000;4:24-30. [PubMed: 10998712] 
9. Fillingim RB, Maixner W, Kincaid S, Silva S. Sex differences in temporal summation but not sensorydiscriminative processing of thermal pain. Pain 1998;75(1):121-127. [PubMed: 9539681]

10. George SZ, Bishop MD, Bialosky JE, Zeppieri GJ, Robinson ME. Immediate effects of spinal manipulation on thermal pain sensitivity: an experimental study. BMC Musculoskel Disord 2006;7 (1):68.

11. Gibson SJ, Helme RD. Age-related differences in pain perception and report. Clin Geriatr Med 2001;17(3):433-456. v-vi. [PubMed: 11459714]

12. Granot M, Friedman M, Yarnitsky D, Zimmer EZ. Enhancement of the perception of systemic pain in women with vulvar vestibulitis. BJOG 2002;109(8):863-866. [PubMed: 12197364]

13. Kirwilliama SS, Derbyshire SWG. Increased bias to report heat or pain following emotional priming of pain-related fear. Pain 2008;137(1):30.

14. Kleinbohl D, Holzl R, Moltner A, Rommel C, Weber C, Osswald PM. Psychophysical measures of sensitization to tonic heat discriminate chronic pain patients. Pain 1999;81:35-43. [PubMed: 10353491]

15. Kosek E, Ordeberg G. Lack of pressure pain modulation by heterotopic noxious conditioning stimulation in patients with painful osteoarthritis before, but not following, surgical pain relief. Pain 2000;88:69-78. [PubMed: 11098101]

16. Martinez V, Fletcher D, Bouhassira D, Sessler DI, Chauvin M. The evolution of primary hyperalgesia in orthopedic surgery: quantitative sensory testing and clinical evaluation before and after total knee arthroplasty. Anesth Analg 2007;105(3):815-821. [PubMed: 17717244]

17. Miron D, Duncan GH, Bushnell MC. Effects of attention on the intensity and unpleastness of thermal pain. Pain 1989;39:345-352. [PubMed: 2616184]

18. Nikolajsen N, Ilkjaer S, Jensen TS. Relationship between mechanical sensitivity and postamputation pain: a prospective study. Eur J Pain 2000;4:327-334. [PubMed: 11124004]

19. Price DD, McGrath PA, Rafii A, Buckingham B. The validation of visual analogue scales as ratio scale measures for chronic and experimental pain. Pain 1983;17:45-56. [PubMed: 6226917]

20. Price DD. Psychological and neural mechanisms of the affective dimension of pain. Science 2000;288 (5472):1769-1772. [PubMed: 10846154]

21. Quiton RL, Greenspan JD. Across- and within-session variability of ratings of painful contact heat stimuli. Pain 2008;137(2):245-256. [PubMed: 17942227]

22. Robinson ME, Wise EA, Gagnon C, Fillingim RB, Price DD. Influences of gender role and anxiety on sex differences in temporal summation of pain. J Pain 2004;5(2):77-82. [PubMed: 15042515]

23. Rosier EM, I MJ, Coghill RC. Reproducibility of pain measurement and pain perception. Pain 2002;98 (1-2):205-216. [PubMed: 12098633]

24. Sarlani E, Greenspan JD. Evidence for generalized hyperalgesia in temporomandibular disorders patients. Pain 2003;102:221-226. [PubMed: 12670662]

25. Schiff E, Eisenberg E. Can quantitative sensory testing predict the outcome of epidural steroid injections in sciatica? A preliminary study. Anesth Analg 2003;97(3):828-832. [PubMed: 12933410]

26. Singer, JD.; Willett, JB. Applied longitudinal data analysis : modeling change and event occurrence. New York:ed. Oxford, NY: Oxford University Press; 2003.

27. Staud R, Vierck CJ, Cannon RL, Mauderli AP, Price DD. Abnormal sensitization and temporal summation of second pain (wind-up) in patients with fibromyalgia syndrome. Pain 2001;91(1-2): 165-175. [PubMed: 11240089]

28. Staud R, Cannon RC, Mauderli AP, Robinson ME, Price DD, Vierck CJ Jr. Temporal summation of pain from mechanical stimulation of muscle tissue in normal controls and subjects with fibromyalgia syndrome. Pain 2003;102(1-2):87-95. [PubMed: 12620600]

29. Staud R, Vierck CJ, Robinson ME, Price DD. Spatial summation of heat pain within and across dermatomes in fibromyalgia patients and pain-free subjects. Pain 2004;111(3):342-350. [PubMed: 15363878]

30. Stratford PW, Goldsmith CH. Use of the standard error as a reliability index of interest: an applied example using elbow flexor strength data. Phys Ther 1997;77(7):745-750. [PubMed: 9225846] 
31. Vase L, Robinson ME, Verne GN, Price DD. Increased placebo analgesia over time in irritable bowel syndrome (IBS) patients is associated with desire and expectation but not endogenous opioid mechanisms. Pain 2005;115(3):338-347. [PubMed: 15911161]

32. Willett JB, Singer JD, Martin NC. The design and analysis of longitudinal studies of development and psychopathology in context: statistical models and methodological recommendations. Dev Psychopathol 1998;10(2):395-426. [PubMed: 9635230]

33. Wright A, Graven-Nielsen T, Davies II, Arendt-Nielsen L. Temporal summation of pain from skin, muscle and joint following nociceptive ultrasonic stimulation in humans. Exp Brain Res 2002;144 (4):475-482. [PubMed: 12037632]

34. Yarnitsky D, Sprecher E, Zaslansky R, Hemli JA. Multiple session experimental pain measurement. Pain 1996;67(2-3):327-333. [PubMed: 8951926]

35. Zautra AJ, Johnson LM, Davis MC. Positive affect as a source of resilience for women in chronic pain. J Consult Clin Psychol 2005;73(2):212-220. [PubMed: 15796628] 


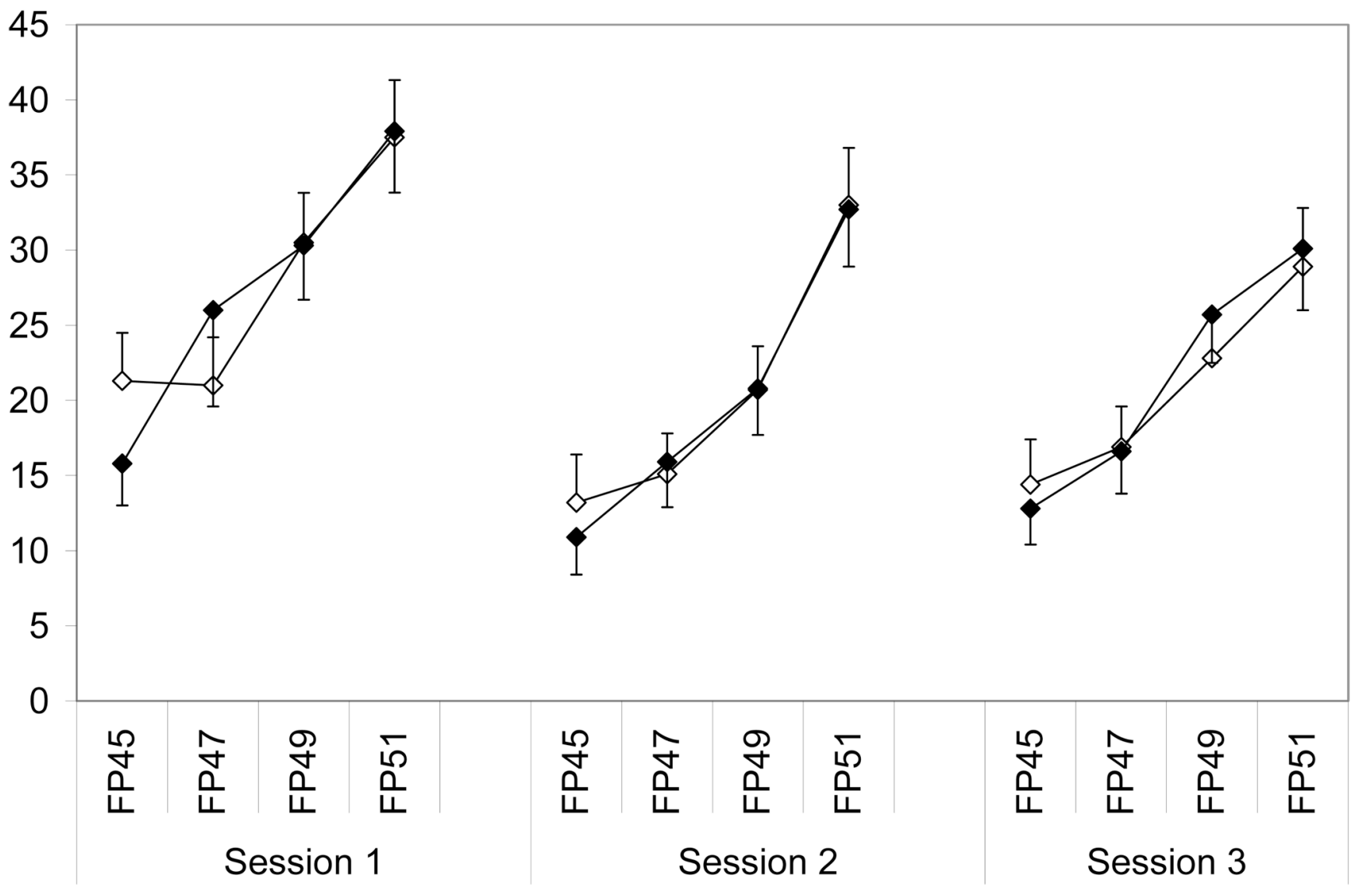

Figure 1.

Ratings of thermally evoked pain at each temperature. Open diamonds are the first set of responses and closed diamonds represented the second set of responses within the same testing session. Session 1 represents the baseline test, session 2 occurred at two weeks and session 3 at four weeks. 




Figure 2.

Negative pain related affect and mean rating of the four thermal stimuli (AvP) Session 1 represents the baseline test, session 2 occurred at two weeks and session 3 at four weeks. 


\section{Table 1}

Mean \pm standard deviation of psychological variables and stimulus parameters the subjects at baseline.

\begin{tabular}{lccc}
\hline & Female $(\mathbf{n = 2 6})$ & Male $(\mathbf{n = 9})$ & $\mathbf{p}$ \\
\hline Session Specific Measures & & & \\
Fear & $13.7 \pm 12.7$ & $16.4 \pm 9.4$ & 0.810 \\
Anxiety & $11.2 \pm 13.1$ & $12.1 \pm 6.5$ & 1.000 \\
Threat & $6.8 \pm 9.1$ & $10.0 \pm 6.8$ & 0.565 \\
Challenge & $16.4 \pm 15.0$ & $20.4 \pm 12.9$ & 0.565 \\
Ratings of thermal stimuli & & & \\
Rating of $45^{\circ} \mathrm{C}$ & $21.9 \pm 20.4$ & $19.0 \pm 13.1$ & 0.028 \\
Rating of $47^{\circ} \mathrm{C}$ & $21.0 \pm 19.2$ & $23.7 \pm 18.3$ & 0.090 \\
Rating of $49^{\circ} \mathrm{C}$ & $31.3 \pm 20.3$ & $28.1 \pm 18.8$ & 0.446 \\
Rating of $51^{\circ} \mathrm{C}$ & $37.8 \pm 22.4$ & $37.3 \pm 21.4$ & 0.271 \\
\hline
\end{tabular}




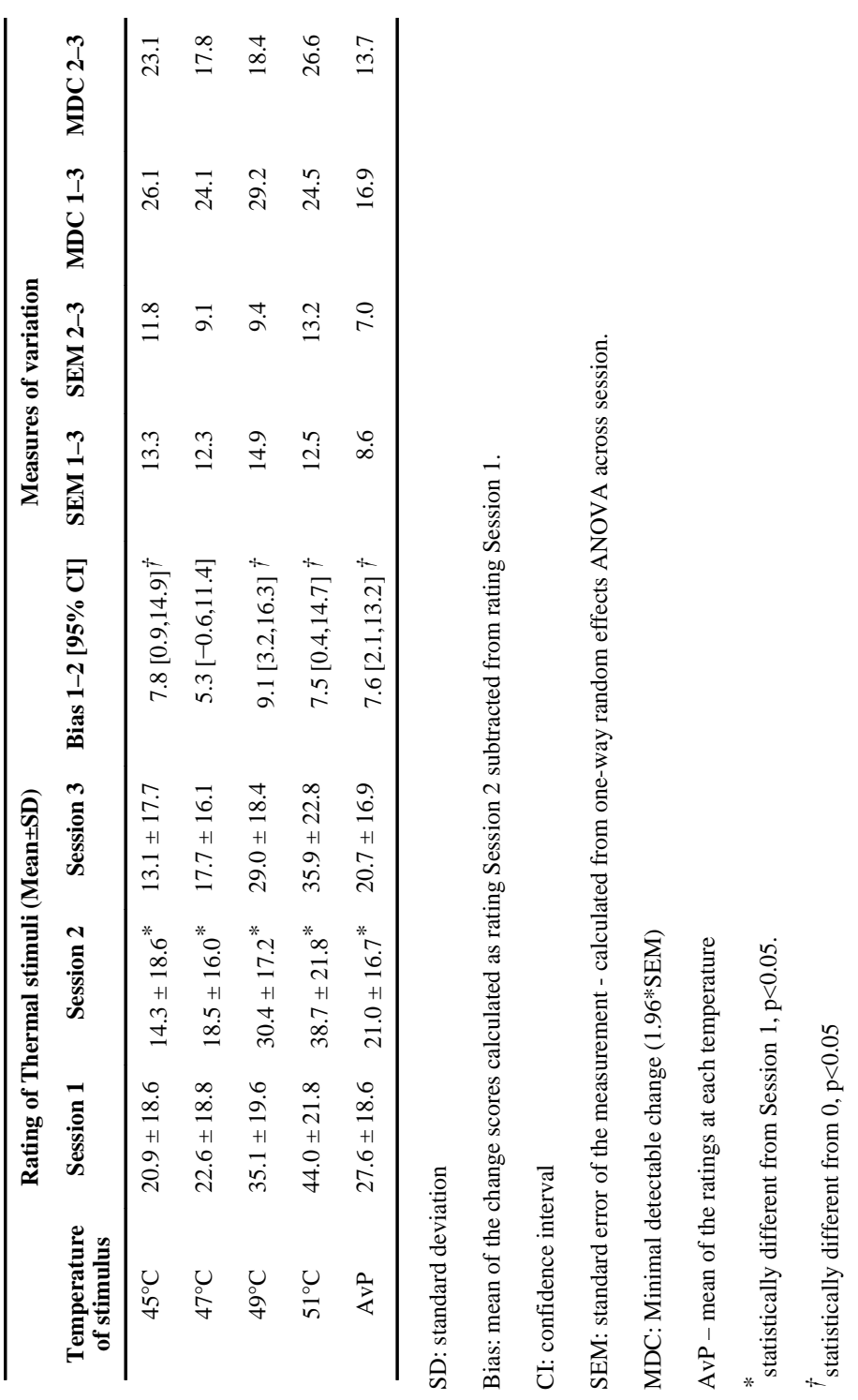

